\title{
FATORES ASSOCIADOS À PRÁTICA DE ATIVIDADE FÍSICA EM HOMENS DE MEIA-IDADE
}

FACTORS ASSOCIATED WITH THE PRACTICE OF PHYSICAL ACTIVITY IN MIDDLE-AGED MEN

FACTORES ASOCIADOS A LA PRÁCTICA DE ACTIVIDAD FÍSICA EN HOMBRES DE MEDIANA EDAD

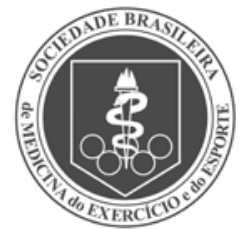

Artigo Original Original ARTICLE Artículo Original
Melissa de Carvalho Souza ${ }^{1}$ (Educadora Física)

Andreia Pelegrini

(Educadora Física)

Marcelo Cozzensa da Silva² (Educador Físico)

Zenite Machado' (Educadora Física)

Adriana Coutinho de Azevedo Guimarães' (Educadora Física)

1. Universidade do Estado de Santa Catarina (UDESC), Florianópolis,

SC, Brasil.

2. Universidade Federal de Pelotas (UFPEL), Pelotas, RS, Brasil.

\section{Correspondência:}

Universidade do Estado de Santa Catarina, Centro de Ciências da Saúde e do Esporte Laboratório de Pesquisa em Lazer e Atividade Física - LAPLAF Rua Pascoal Simone, 358, Coqueiros, Florianópolis. CEP: 88080-350 mecarvalho.s@gmail.com

\section{RESUMO}

Introdução: O aumento dos níveis de atividade física (AF) tem sido importante para uma vida saudável, e identificar os fatores que se associam a essa prática pode incentivar a adoção de um estilo de vida ativo. Objetivo: Analisar os fatores associados à prática de AF atual em homens de meia-idade. Métodos: Estudo transversal com amostra probabilística de 416 homens de meia-idade ( 40 a 59 anos) em duas cidades no sul do Brasil. Para a coleta de dados utilizou-se um questionário dividido em seis partes: características da amostra; medidas antropométricas; estrato econômico - IBGE; AF - IPAQ-versão curta; percepção de saúde - BRFSS; e sintomas do envelhecimento masculino - escala dos sintomas do envelhecimento masculino (AMS). A amostra foi dividida em dois grupos etários: 40 a 49 e 50 a 59 anos. A análise estatística foi descritiva e inferencial. Resultados: Aproximadamente 60,2\% dos homens foram considerados suficientemente ativos (ativos + muito ativos), entretanto, não foram observadas diferenças significativas entre as faixas etárias $(p=0,152)$. A caminhada foi a AF mais prevalente e a atividade vigorosa, a menos prevalente. As intensidades da AF (caminhada moderada e vigorosa) se relacionaram com a escolaridade, classe econômica, percepção de saúde e sintomas do envelhecimento masculino. Os homens acima do peso e com sintomas apresentaram probabilidade maior ( $R P=1,12$; IC 95\% =1,01-1,24; RP = 1,30; IC $95 \%=1,00-1,69)$ de ter AF insuficiente. Conclusão: O presente estudo identificou a relação de determinadas características pessoais dos participantes com a intensidade da AF praticada. Além disso, foi possível identificar associação entre sintomas do envelhecimento masculino e condições de peso e o nível de AF dos homens de meia-idade. Assim, estes resultados podem atuar como insumo à promoção da AF para homens nessa faixa etária, como forma a melhorar o estilo de vida e os cuidados com a saúde.

Descritores: atividade motora; homens; saúde.

\section{ABSTRACT}

Introduction: Increased levels of physical activity (PA) have been important for a healthy life, and to identify associated factors with this practice can encourage the adoption of an active lifestyle. Objective: To analyze the factors associated with the current practice of PA in middle-aged men. Methods: Cross-sectional study with a random sample of 416 middle-aged men (40 to 59 years) in two cities in southern Brazil. A questionnaire was used for data collection, divided into six parts: sample characteristics; anthropometric measurements; socioeconomic strata - IBGE; PA - IPAQ-short version; health perception - BRFSS, and aging male symptoms by the Aging Males' Symptoms scale (AMS). The sample was divided into two age groups: from 40 to 49, and 50 to 59 years old. The statistical analysis was descriptive and inferential. Results: Approximately $60.2 \%$ of men were considered sufficiently active (active + very active), however, there were no significant differences between the age groups ( $p=0.152)$. Walking was the most prevalent $P A$ and vigorous activity the least prevalent. The intensities of PA (moderate and vigorous walking) were related to education, socioeconomic status, health perception, and symptoms of male aging. The overweight men and with symptoms were more likely of having insufficient $P A(O R=1.12 ; 95 \% \mathrm{Cl}=1.01-1.24 ; \mathrm{OR}=1.30 ; 95 \% \mathrm{Cl}=1.00-1.69)$. Conclusion: This study identified the relationship of certain personal characteristics of the participants with the intensity of PA. Moreover, it was possible to identify an association between symptoms of male aging and weight status and the level of PA of middle-aged men. Thus, these findings can serve as input to promote PA for men in this age group as a way to improve the life style and care for health.

Keywords: motor activity; men; health.

\section{RESUMEN}

Introducción: El aumento de los niveles de actividad física (AF) ha sido importante para una vida sana, e identificar los factores que están asociados con esta práctica puede estimular la adopción de un estilo de vida activo. Objetivo: Analizar los factores asociados con la práctica actual de la AF en los hombres de mediana edad. Métodos: Estudio transversal con una muestra aleatoria de 416 hombres de mediana edad (40-59 años) en dos ciudades en el sur de Brasil. Para la recolección de datos se utilizó un cuestionario dividido en seis partes: características de la muestra; medidas antropométricas; estratos económicos - IBGE; AF-IPAQ, versión corta; percepción de salud - BRFSS y sintomas de envejecimiento masculino - escala de síntomas del envejecimiento masculino (AMS). La muestra se dividió en dos grupos: de 40 a 49 y 50 a 59 años de edad. El análisis estadístico fue descriptivo e inferencial. Resultados: Aproximadamente el 60,2\% de los hombres se consideraron suficientemente activos (activos + muy activos), 
sin embargo, no hubo diferencias significativas entre los grupos de edad ( $p=0,152)$. La caminata fue el AF más frecuente y la actividad vigorosa fue la menos frecuente. Las intensidades de AF (caminata moderada y vigorosa) fueron relacionadas con educación, situación económica, percepción de la salud y síntomas del envejecimiento masculino. Los hombres con sobrepeso y con síntomas fueron más propensos ( $R R=1,12 ; / C 95 \%: 1,01-1,24 ; R R=1,30$; IC 95\%: 1,00-1,69) a presentar AF insuficiente. Conclusión: Este estudio identificó la relación de ciertas características personales de los participantes con la intensidad de AF practicada. Además fue posible identificar una asociación entre sintomas del envejecimiento masculino y el peso con el nivel de AF de los hombres de mediana edad. Por lo tanto, estos resultados pueden actuar como incentivo para promover AF para los hombres en este grupo de edad, como una forma de mejorar el estilo de vida y el cuidado de salud.

\section{Descriptores: actividad motora; hombres; salud.}

\section{INTRODUÇÃO}

O aumento dos níveis de AF é reconhecido como uma das mais importantes formas para manter uma vida saudável ${ }^{1,2}$. A AF não trata somente de atividades sistematizadas, pode ser praticada no lazer, no trabalho, em tarefas domésticas e no deslocamento, em intensidades moderadas e/ou vigorosas ${ }^{3}$.

Verifica-se um crescente interesse de diferentes grupos populacionais na prática de $\mathrm{AF}^{4}$, visando a prevenção e a promoção da saúde, incluindo a preocupação com um envelhecimento mais saudável ${ }^{4}$. De acordo com Del Duca et al. ${ }^{3}$, no Brasil, aproximadamente 41,2\% dos homens são ativos, enquanto nas mulheres este número é de 27,4\%. Embora essa população apresente maiores níveis de AF em comparação às mulheres, é notável que na maior parte dos casos, são os homens que apresentam maior incidência de doenças crônicas, e com o avanço da idade, taxa de mortalidade mais elevada 5 .

Há um crescente interesse em estudar a população masculina, todavia são escassos os estudos que investigam a AF em homens na meia idade (40 a 59 anos). Os estudos com homens nesta faixa etária, se concentram em países da América do Norte, Europa e Oceania ${ }^{6,7}$.

Considerando ser uma população que se prepara para o processo do envelhecimento, e pode enfrentar sintomas somáticos, psicológicos e sexuais, caracterizando os sintomas do envelhecimento masculino, é imperativo o estudo da AF, como forma de auxiliar a experenciar este processo natural de forma menos danosa, à saúde ${ }^{8}$. Além disso, identificar os fatores que se associam à AF em homens, pode ser um determinante ao incentivo de sua prática em diferentes intensidades, faixas etárias e condições de saúde. Deste modo, este estudo objetivou analisar os fatores associados à prática de AF atual em homens na meia idade.

\section{METODOLOGIA}

O estudo exploratório observacional analítico com delineamento transversal, faz parte de uma investigação mais abrangente a fim de analisar a prevalência dos sintomas do envelhecimento masculino e sua associação com a AF e qualidade de vida em homens de meia idade. Este estudo foi aprovado pelo Comitê de Ética em Pesquisa em Seres Humanos da Universidade do Estado de Santa Catarina, protocolo n. 535.729/2014.

A população foi composta por homens na meia idade ${ }^{9}$, da Região da Grande Florianópolis, Sul do Brasil. Foram selecionadas as cidades dessa Região com mais de 200 mil habitantes com características econômicas semelhantes, segundo o último Censo ${ }^{10}$, nomeadamente, Florianópolis e São José, com uma população masculina na meia idade de 51.740 e 20.939, respectivamente. Assim a população foi constituída de 72.679 homens, estratificada em nível socioeconômico a partir do IBGE ${ }^{10}$, tendo como base o salário mínimo (SM) do ano de 2014 de R\$ 724,00.
Para estabelecer o tamanho da amostra ${ }^{11}$ adotou-se uma prevalência de $16 \%$ de prática de AF para homens na meia idade ${ }^{12}$, erro tolerável de quatro pontos percentuais, nível de confiança de 95\%, efeito de delineamento de 1,5. Foi acrescido 10\% para possíveis perdas e recusas, sendo a amostra final pretendida de 530 homens. Proporcionalmente a cada classe econômica obteve-se 26 homens da classe A, 163 da classe B, 172 da classe C e 169 da classe D. Os bairros classe E não foram caracterizados pelo censo por serem setores com baixa densidade de domicílios ${ }^{10}$.

Foram excluídos indivíduos que apresentaram as seguintes doenças: câncer, doenças cardiovasculares, diabetes, hipertensão arterial e paraplegias, por se tratarem de doenças que influenciam diretamente na ocorrência dos sintomas do envelhecimento masculino. Também foram excluídos indivíduos institucionalizados e com incapacidade para compreender o questionário.

Para este estudo foram contatados 1.150 domicílios. Em 338 não haviam homens na meia idade, e dos domícilios nos quais residiam homens nesta faixa etária 396 se recusaram a fazer parte do estudo. Assim, a amostra final por conglomerado aleatória foi de 416 homens com média de idade de 49 6 anos, sendo 24 da classe A, 89 da classe B, 234 da classe C e 69 da classe D, representando 78\% da amostra necessária de acordo com o cálculo realizado.

Para a coleta de dados foi utilizado um questionário padronizado composto por instrumentos validados dividido em seis blocos descritos a seguir:

Informações referentes à idade, situação conjugal, escolaridade, presença de doença diagnosticada, utilização de medicamentos para doença e para desempenho sexual. A idade foi estratificada em: 40 a 49 e 50 a 59 anos.

Peso corporal e estatura auto-referidos ${ }^{13}$ foram coletados para o cálculo do Índice de Massa Corporal - IMC. Utilizou-se o protocolo da Organização Mundial da Saúde ${ }^{14}$. As categorias foram agrupadas em: peso normal e acima do peso.

Foi obtida segundo IBGE ${ }^{10}$ classificando a população em estratos econômicos $A, B, C, D$ e $E$, por meio da renda familiar média mensal. Os estratos foram agrupados em classe alta (A e B); classe média (C) e classe baixa (D e E).

Foram avaliados pela escala dos sintomas do envelhecimento masculino - AMS $^{15}$. O somatório geral das questões, foi considerado como o escore geral, sendo dicotomizado em: $\geq 27$ pontos, com sintomas do envelhecimento, e valores inferiores a esse sem sintomas.

Por meio do Behavioral Risk Factors Surveillance System Questionaire $^{16}$. As respostas são em uma escala "likert", 1 excelente; 2 muito boa; 3 boa; 4 regular; 5 ruim. As respostas 1 a 3 foram agrupadas em muito boa e as respostas 4 e 5 em ruim.

O Questionário Internacional de AF - IPAQ (versão curta) ${ }^{17}$ foi utilizado para avaliar o nível de AF. Quantificou-se o número de vezes 
de atividades realizadas por no mínimo 10 minutos contínuos de caminhada, intensidade moderada e vigorosa na última semana, no âmbito, ocupacional, doméstico, no lazer e deslocamento. A soma da duração das diferentes intensidades foi realizada para estabelecer os critérios: ativo - cumpriu com as recomendações de AF vigorosa $\geq 3$ dias/semana e $\geq 20$ minutos/sessão; moderada $\geq 5$ dias/semana e $\geq$ 30 minutos /sessão; qualquer atividade somada: $\geq 5$ dias/semana e $\geq$ $150 \mathrm{~min} / \mathrm{semana}$. Muito ativo: vigorosa $\geq 5$ dias/semana e $\geq 30 \mathrm{~min} /$ sessão; vigorosa $\geq 3$ dias/semana e $\geq 20 \mathrm{~min} /$ sessão + moderada $\geq$ 5 dias/semana e $\geq 30 \mathrm{~min} / \mathrm{sessão.} \mathrm{Os} \mathrm{indivíduos} \mathrm{que} \mathrm{não} \mathrm{cumpriram}$ com estes critérios foram classificados como insuficientemente ativos.

A coleta de dados foi realizada por uma equipe composta por sete indivíduos de ambos os sexos, com idade superior a 18 anos, com pelo menos, ensino médio completo, os quais receberam instruções prévias da pesquisadora principal do estudo.

Foi realizado o sorteio dos CEPs proporcionalmente às quatro classes econômicas dos bairros de Florianópolis e São José. Os CEPs de cada bairro foram catalogados em planilha eletrônica no programa Excel versão 2010, também foi utilizado para o sorteio.

Após este procedimento percorreu-se a rua correspondente ao CEP sorteado e selecionado o primeiro domicílio da referida rua para incluir no estudo. $O$ intervalo entre as casas foi conforme a quantidade de domicílios em cada rua nos quais residissem homens de meia idade. O domicílio foi excluído após a terceira tentativa de contato (perda), e quando o indivíduo recusava-se a participar do estudo. Este processo foi replicado em todas as classes econômicas.

O questionário foi aplicado em formato de entrevista. No bloco dos sintomas sexuais da escala dos sintomas do envelhecimento masculino o participante optava em responder individualmente ou prosseguir com a entrevista.

Para amenizar perdas amostrais e evitar constrangimento, quando solicitado pelo participante na visita ao seu domicílio foi realizado contato telefônico e obtido o endereço de e-mail, pois os mesmos alegavam se sentir à vontade em preecher o questionário dessa maneira.

Todos os participantes que concordaram em participar do estudo após explicação dos objetivos do mesmo, assinaram o termo de consentimento livre e esclarecido.

A análise estatística foi realizada pelo Statistical Package for Social Sciences (SPSS) - versão 20.0. Utilizou-se a estatística descritiva (média, desvio padrão e percentual). A normalidade dos dados foi verificada por meio do teste Kolmogorov-Smirnov. Para as associações das características pessoais e nível de AF entre os grupos etários, utilizou-se o teste do Qui-quadrado. A comparação entre intensidades de AF e características pessoais foi efetuada pelos testes $U$ de Mann-Whitney e Kruskal-Wallis.

A associação entre o desfecho AF e as variáveis independentes foi analisada por meio da regressão de Poissson. Foi testado um modelo simples e outro múltiplo (ajustado para todas as variáveis) ${ }^{18}$. Em todas as análises adotou-se o nível de significância de 5\%.

\section{RESULTADOS}

As características dos participantes de acordo com a faixa etária, são apresentadas na tabela 1. Não foi observada diferença significativa, todavia verificou-se que a maioria dos homens apresentava ensino superior completo $(55,8 \%)$ com média de $15 \pm 7$ anos de estudo. Pertenciam a classe econômica alta (37,7\%), e moravam com companheira (81,8\%). Não apresentavam doenças diagnosticadas (81,7\%), não utilizavam medicamentos para doenças e para desempenho sexual (88,2\%; 98,3\%). Quanto a percepção de saúde a consideravam como muito boa $(82,9 \%)$ e a maioria $(61,4 \%)$ relatou a presença de sintomas do envelhecimento masculino. Com referência ao IMC, apresentaram uma média de $27 \pm 4 \mathrm{~kg} / \mathrm{m}^{2}$.

Não foram observadas diferenças significativas no nível de AF de acordo com os grupos etários (Figura 1). Entretanto, observa-se que a maioria dos homens apresentaram níveis suficientes de AF (60,2\%), sendo que aqueles de 40-49 anos demonstraram um nível de AF levemente superior (63,9\%).

A Figura 2 mostra a distribuição das intensidades de AF por faixa etária. A caminhada foi a atividade mais praticada entre todas as faixas etárias ( 40 minutos/dia), seguida da AF moderada ( 30 minutos/ dia), e por fim a AF vigorosa ( 20 minutos/dia). Não houve diferença significativa entre os grupos.

Tabela 1. Características da amostra de acordo com a faixa etária dos homens participantes do estudo (\%). Florianópolis e São José, SC. 2014. ( $n=416)$.

\begin{tabular}{|c|c|c|c|c|}
\hline & Total & 40-49 anos & 50-59 anos & p valor \\
\hline Escolaridade & & & & 0,113 \\
\hline Ensino fundamental & 12,8 & 9,3 & 15,8 & \\
\hline Ensino médio & 31,3 & 34,0 & 29,0 & \\
\hline Ensino superior & 55,9 & 56,7 & 55,2 & \\
\hline Classe econômica & & & & 0,201 \\
\hline Baixa & 27,5 & 30,1 & 25,3 & \\
\hline Média & 34,8 & 36,8 & 33,3 & \\
\hline Alta & 37,7 & 33,2 & 41,6 & \\
\hline Estado conjugal & & & & 0,479 \\
\hline Sem companheira & 18,2 & 19,6 & 16,9 & \\
\hline Com companheira & 81,8 & 80,4 & 83,1 & \\
\hline Presença de doença & & & & 0,056 \\
\hline Sim & 18,3 & 14,4 & 21,7 & \\
\hline Não & 81,7 & 85,6 & 78,3 & \\
\hline Medicamento doença & & & & 0,072 \\
\hline Sim & 11,8 & 8,8 & 14,5 & \\
\hline Não & 88,2 & 91,2 & 85,5 & \\
\hline Medicamento sexual & & & & $0,085 \#$ \\
\hline Sim & 1,7 & 0,5 & 2,7 & \\
\hline Não & 98,3 & 99,5 & 97,3 & \\
\hline Percepção de saúde & & & & 0,405 \\
\hline Ruim & 17,1 & 15,5 & 18,6 & \\
\hline Muito boa & 82,9 & 84,5 & 81,4 & \\
\hline $\begin{array}{c}\text { Sintomas do envelhecimento } \\
\text { masculino }\end{array}$ & & & & 0,395 \\
\hline Sem sintomas & 38,6 & 40,7 & 36,7 & \\
\hline \multirow[t]{2}{*}{ Com sintomas } & 61,4 & 59,3 & 63,3 & \\
\hline & $\mathbf{X} \square(\mathrm{dp})$ & $\mathbf{x} \square(\mathrm{dp})$ & $\mathbf{x} \square(\mathrm{dp})$ & \\
\hline $\mathrm{IMC} * *$ & $27(4)$ & $27(4)$ & $27(3)$ & 0,869 \\
\hline Anos de estudo & $15(7)$ & $16(7)$ & $15(8)$ & 0,777 \\
\hline
\end{tabular}

Teste do qui-quadrado; \# Exato de fischer; **índice de massa corporal.

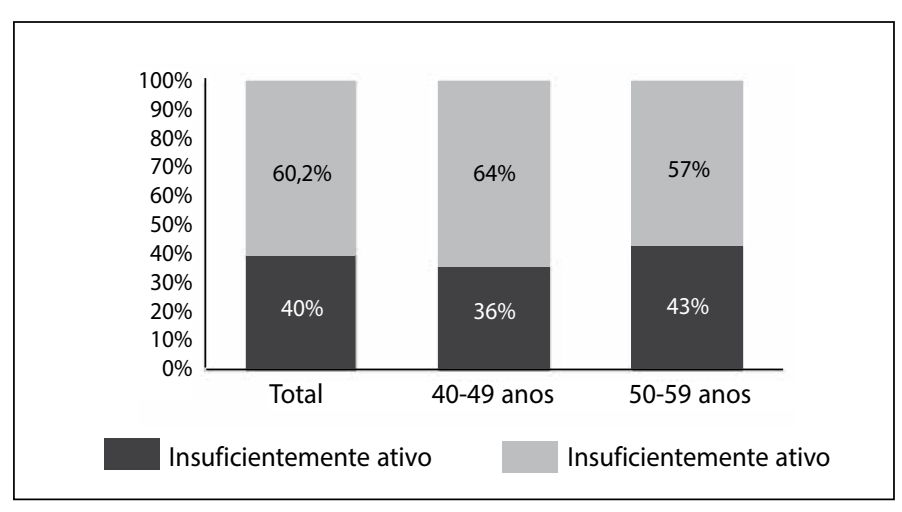

Figura 1. Nível de AF atual de acordo com a faixa etária dos homens participantes do estudo (\%). Florianópolis e São José, SC. 2014. $(n=416)$ - $p=0,152$. 
A comparação entre a intensidade da AF e as variáveis independentes, é demonstrada na tabela 2. A variável que apresentou diferença significativa em relação à caminhada foi aquela relacionada aos sintomas do envelhecimento masculino ( $p=0,038)$, os homens sem sintomas apresentaram um tempo de caminhada mais elevado. Na prática de AF moderada, aqueles com ensino superior $(p=0,010)$, classe econômica média e alta $(p=0,032)$ e sem sintomas do envelhecimento masculino $(p=0,008)$ foram mais ativos. Na AF vigorosa, os que consideraram sua saúde como muito boa apresentaram médias superiores $(p=0,004)$.

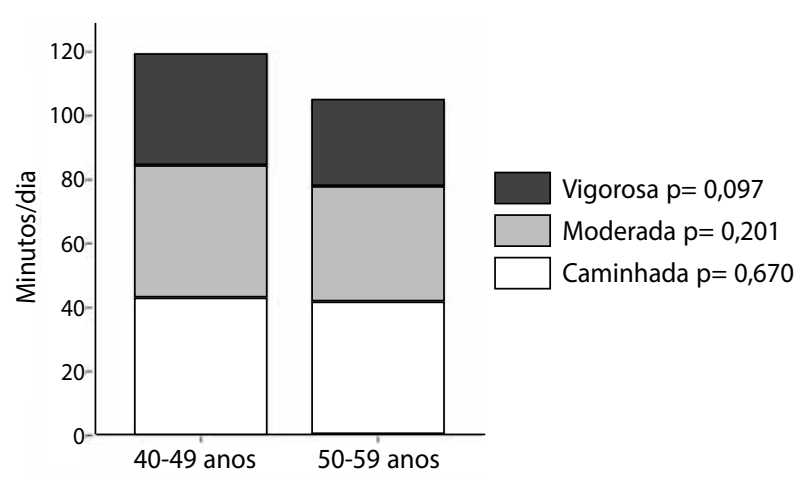

Figura 2. Caminhada, AF moderada e vigorosa de acordo com a faixa etária dos homens participantes do estudo. Florianópolis e São José, SC. 2014. (n=416).
A tabela 3 apresenta as associações do nível de AF com as variáveis independentes. No modelo simples o nível de AF associou-se com status de peso e sintomas do envelhecimento masculino. Quando o modelo foi ajustado para todas as variáveis, as associações foram mantidas. Os homens acima do peso e com sintomas apresentaram uma probabilidade maior $(\mathrm{RP}=1,12$; IC95\%=1,01-1,24; $\mathrm{RP}=1,30$; $\mathrm{IC} 95 \%=$ 1,00-1,69), de serem insuficientemente ativos.

\section{DISCUSSÃO}

As recomendações mundias de AF preconizam que o indivíduo para ser considerado suficientemente ativo deve acumular pelo menos 150 minutos de AF moderada por semana, ou 75 minutos de AF vigorosa ${ }^{19}$. Os resultados do presente estudo identificaram que os homens foram suficientemente ativos, por apresentarem, níveis superiores às recomendações supracitadas. É possível que este resultado seja em virtude de algumas características pessoais dos participantes, como o elevado grau de escolaridade e classe econômica de média à alta, pois de acordo com a literatura, indivíduos com maior grau de escolaridade apresentam em geral níveis mais elevados de AF devido à distinções socioeconômicas ${ }^{20}$.

Confirmando tal constatação, os resultados deste estudo apontaram que os homens com ensino superior e pertencentes às classes econômicas média e alta, apresentaram um tempo de prática de AF moderada superior aos seus pares. Semelhantemente, outro estudo sugere que homens com elevados níveis de escolaridade apresen-

Tabela 2. Comparação entre intensidades da AF atual e as vatiáveis indenpendentes dos homens participantes do estudo. Florianópolis e São José, SC. 2014. (n=416).

\begin{tabular}{|c|c|c|c|c|c|c|}
\hline Variáveis & $\begin{array}{c}\text { Caminhada** } \\
\mathbf{X} \square \text { (dp) }\end{array}$ & $p$ valor & $\begin{array}{c}\text { Moderada** } \\
\quad \mathrm{x} \square(\mathrm{dp})\end{array}$ & p valor & $\begin{array}{c}\text { Vigorosa** } \\
\mathrm{X} \square(\mathrm{dp})\end{array}$ & $p$ valor \\
\hline Faixa etária* & & 0,670 & & 0,201 & & 0,097 \\
\hline 40-49 anos & $42(44)$ & & $41(48)$ & & $35(47)$ & \\
\hline 50-59 anos & $41(44)$ & & $36(45)$ & & $28(40)$ & \\
\hline Escolaridade\# & & 0,124 & & 0,010 & & 0,474 \\
\hline Ensino fundamental & $39(50)$ & & $28(47)$ & & $33(57)$ & \\
\hline Ensino médio & $50(51)$ & & $39(53)$ & & $30(45)$ & \\
\hline Ensino superior & $38(37)$ & & $40(42)$ & & $31(40)$ & \\
\hline Classe econômica\# & & 0,911 & & 0,032 & & 0,371 \\
\hline Baixa & $49(57)$ & & $34(51)$ & & $29(49)$ & \\
\hline Média & $38(37)$ & & $41(50)$ & & $35(47)$ & \\
\hline Alta & $39(38)$ & & $39(38)$ & & $29(37)$ & \\
\hline Estado conjugal* & & 0,867 & & 0,304 & & 0,273 \\
\hline Sem companheira & $41(40)$ & & $44(51)$ & & $25(36)$ & \\
\hline Com companheira & $42(45)$ & & $37(45)$ & & $33(45)$ & \\
\hline Presença de doença* & & 0,635 & & 0,853 & & 0,062 \\
\hline $\operatorname{Sim}$ & $42(47)$ & & $39(47)$ & & $24(39)$ & \\
\hline Não & $42(44)$ & & $38(46)$ & & $33(45)$ & \\
\hline Medicamento doença* & & 0,216 & & 0,644 & & 0,053 \\
\hline Sim & $39(49)$ & & $39(52)$ & & $22(38)$ & \\
\hline Não & $42(43)$ & & $38(45)$ & & $33(44)$ & \\
\hline Medicamento sexual* & & 0,528 & & 0,576 & & 0,939 \\
\hline $\operatorname{sim}$ & $54(59)$ & & $24(25)$ & & $31(46)$ & \\
\hline Não & $41(44)$ & & $39(46)$ & & $31(44)$ & \\
\hline Status de peso* & & 0,641 & & 0,086 & & 0,079 \\
\hline Peso normal & $43(45)$ & & $46(53)$ & & $37(48)$ & \\
\hline Acima do peso & $41(44)$ & & $35(43)$ & & $29(42)$ & \\
\hline Percepção de saúde* & & 0,071 & & 0,845 & & 0,004 \\
\hline Ruim & $37(47)$ & & $38(46)$ & & $21(42)$ & \\
\hline Muito boa & $43(44)$ & & $38(46)$ & & $33(44)$ & \\
\hline Sintomas do envelhecimento masculino* & & 0,038 & & 0,008 & & 0,352 \\
\hline Sem sintomas & $49(50)$ & & $46(49)$ & & $34(46)$ & \\
\hline Com sintomas & $37(40)$ & & $34(43)$ & & $30(42)$ & \\
\hline
\end{tabular}


Tabela 3. Análise de regressão bruta e ajustada entre nível de AF atual e as variáveis independentes. Florianópolis e São José, SC. 2014. (n=416).

\begin{tabular}{|c|c|c|c|c|}
\hline Variáveis & $\begin{array}{l}\text { RP* bruta } \\
\text { (IC95\%) }\end{array}$ & p valor & $\begin{array}{l}\mathrm{RP}^{*} \text { ajustada } \\
\text { (IC95\%) }\end{array}$ & $p$ valor \\
\hline Faixa etária & & 0,150 & & \\
\hline 40-49 anos & 1 & & - & \\
\hline 50-59 anos & $1,07(0,98-1,18)$ & & - & \\
\hline Escolaridade & & 0,660 & & \\
\hline Ensino fundamental & 1 & & & \\
\hline Ensino médio & $1,03(0,89-1,21)$ & & - & \\
\hline Ensino superior & $1,01(0,88-1,16)$ & & - & \\
\hline Classe econômica & & 0,265 & & \\
\hline Baixa & 1 & & & \\
\hline Média & $1,07(095-1,21)$ & & - & \\
\hline Alta & $1,05(0,93-1,18)$ & & - & \\
\hline Estado conjugal & & 0,853 & & \\
\hline Sem companheira & 1 & & & \\
\hline Com companheira & $1,01(0,90-1,14)$ & & - & \\
\hline Presença de doença & & 0,493 & & \\
\hline Sim & 1 & & & \\
\hline Não & $0,96(0,85-1,08)$ & & - & \\
\hline Medicamento doença & & 0,175 & & \\
\hline $\operatorname{Sim}$ & 1 & & - & \\
\hline Não & $0,90(0,78-1,05)$ & & - & \\
\hline Medicamento sexual & & 0,353 & & \\
\hline $\operatorname{Sim}$ & 1 & & - & \\
\hline Não & $0,84(0,58-1,21)$ & & - & \\
\hline Status de peso & & 0,015 & & 0,030 \\
\hline Peso normal & 1 & & 1 & \\
\hline Acima do peso & $1,13(1,03-1,25)$ & & $1,12(1,01-1,23)$ & \\
\hline Percepção de saúde & & 0,081 & & \\
\hline Ruim & 1 & & - & \\
\hline Muito boa & $0,89(0,79-1,01)$ & & - & \\
\hline $\begin{array}{c}\text { Sintomas do envelhecimento } \\
\text { masculino }\end{array}$ & & 0,023 & & 0,049 \\
\hline Sem sintomas & 1 & & 1 & \\
\hline Com sintomas & $1,12(1,01-1,23)$ & & $1,30(1,00-1,69)$ & \\
\hline
\end{tabular}

*RP: Razăo de prevalência.

tam uma chance 1,6 (IC95\%= 1,3-2,0) vezes maior de serem ativos no lazer ${ }^{21}$. Há evidências de que baixos níveis de escolaridade e renda estão relacionados a uma chance diminuida no engajamento em AF moderadas à vigorosas e de lazer22.

Em relação a intensidade de AF praticada neste estudo, curiosamente ambos os grupos etários apresentaram maior prevalência em atividades de caminhada, seguida da atividade moderada, enquanto é observado na literatura que os homens, usualmente, praticam atividades vigorosas ${ }^{23}$. Entretanto deve-se levar em conta, que os resultados na literatura consideram amostras com idade de até 35 anos, na qual há elevada prática de AF principalmente no lazer, com atividades como futebol e musculação. Ao passar dos anos o interesse por tais atividades se modifica, preferindo-se atividades mais leves, como a caminhada, sendo a mais comum em populações com idade superior a 40 anos ${ }^{23-25}$.

Nesta perspectiva, considerando a intensidade da AF, neste estudo identificou-se influência da percepção de saúde. Os homens que consideraram sua saúde como muito boa apresentaram um tempo de prática de AF vigorosa superior. É importante relatar, que a AF vigorosa pode corresponder a uma redução do tempo para a prevenção de doenças e promover a longevidade, contribuindo para uma melhor percepção de saúde ${ }^{26}$.

Além deste fator foi identificado a relação da prática de caminhada e de AF moderada com os sintomas do envelhecimento masculino. Os homens sem os sintomas praticavam maior tempo de caminhada e atividade moderada. Ainda observou-se associação dos sintomas com o nível de AF. Os indivíduos com sintomas do envelhecimento masculino apresentaram uma probabilidade maior de serem insuficientemente ativos.

O aumento dos níves de AF proporciona benefícios para os aspectos somáticos, psicológicos e sexuais, de uma forma geral. É possível que a mesma influencie na menor prevalência dos sintomas, haja vista que a sintomatologia geral do envelhecimento masculino é decorrente destes aspectos ${ }^{8}$. Assim, menores nível de AF pode contribuir para maior ocorrência da sintomatologia.

Nossos resultados indicaram ainda que os homens acima do peso apresentaram maiores chances de serem insuficientemente ativos. Estes resultados vão ao encontro do que é relatado em homens na meia idade, quando se comprova a existência de influência do peso corporal para a adesão de $\mathrm{AF}^{27}$. Pode-se dizer, que o excesso de peso tem sido um fator determinante para comportamentos inadequados de AF, além de ser um fator de risco para o desenvolvimento de doenças crônico-degenerativas ${ }^{27}$. Considera-se que a insatisfação com o excesso de peso pode afetar negativamente a motivação para a prática de $\mathrm{AF}^{25}$, uma vez que, em geral, indivíduos nesta condição não se envolvem em AF em grupo por sentirem constrangimento de se expor em atividades corporais ${ }^{28,29}$.

É importante citar, que este estudo apresenta limitações, como o número de perdas e recusas, devido a dificuldade no acesso aos homens em suas residências. Ainda a avaliação da AF por informação autorelatada, pode superestimar este comportamento por ser uma prática desejável. Acredita-se que a utilização do IPAQ em sua versão longa seria uma melhor forma para mensuração da AF, pois o IPAQ utilizado considera os quatro domínios da AF em conjunto, assim os indivíduos tendem a não fornecer respostas precisas ${ }^{30}$. Entretanto é um questionário extenso, dificultando a obtenção dos dados nesta população, pela relutância dos homens em participar de pesquisas. Também sendo um estudo de corte transversal, impede constatações de causa e efeito.

No que se refere às vantagens, relaciona-se principalmente à utilização de um desenho amostral probabilístico, o qual permitiu a aleatorização dos sujeitos, sendo que todos os indíviduos da população tiveram a mesma probabilidade em serem incluídos no estudo. O fato de investigar uma população ainda pouco estudada no Brasil, pode possibilitar um maior conhecimento do estilo de vida especificamente em homens na meia idade, bem como os fatores que se associam à AF neste período.

\section{CONCLUSÃO}

O presente estudo identificou a prática de AF de homens na meia idade, e fatores a ela associados. Estes resultados podem contribuir para o insumo de políticas públicas de promoção à saúde, com o incentivo ao aumento da prática de AF para homens nessa faixa etária. Contudo, ressalta-se a importância de investigações na área, com delineamentos longitudinais e/ou experimentais, afim de verificar possíveis relações causais com a prática de AF.

Todos os autores declararam não haver qualquer potencial conflito de interesses referente a este artigo. 
CONTRIBUIÇÕES DOS AUTORES: MCS (0000-0002-7861-7620)* foi responsável pela concepção, delineamento, coleta, análise e interpretação dos dados; redação do artigo e aprovação da versão submetida. AP (0000-0001-8862-9636)* e MCS (0000-0003-2336-7131)* se dedicaram a análise e interpretação dos dados; redação do artigo e aprovação da versão submetida. ZM (0000-0002-4569-8460)* foi responsável pela redação do artigo e aprovação da versão submetida. ACAG (0000-00015167-2921)* pela concepção, delineamento, coleta, análise e interpretação dos dados; redação do artigo e aprovação da versão submetida. *Número ORCID (Open Researcher and Contributor ID).

\section{REFERÊNCIAS}

1. Guallar-Castillón P, Bayan-Bravo A, León-Muñoz LM, Balboa-Castillo T, López-García JL, Rodríguez-Artalejo F, et al. The association of major patterns of physical activity, sedentary behavior and sleep with health-related quality of life: a cohort study. Prev Med. 2014;67:248-54.

2. Carlier M, Delevoye-Turrell Y, Dione M. Cognitive Benefits of Physical Activity Increased when Producing Rhythmic Actions. Proc Soc Behav Sci. 2014;126:235-6.

3. Del Duca GF, Nahas MV, Hallal PC, Peres KG. Atividades físicas no lazer entre adultos de Florianópolis, Santa Catarina, Brasil: estudo populacional sobre as características das práticas e de seus praticantes. Ciênc Saúde Coletiva. 2014;19(11):4595-604.

4. Brasil. Ministério da saúde. Braślila. Aumento na prática de atividades físicas, 2014 [acesso em 2015 jan 13]. Disponível em: http://www.brasil.gov.br.

5. Department of Health and Ageing. National male health policy: Building on the strengths of Australian males. Canberra: Department of Health and Ageing; 2010

6. Dixon NC, Hurst TL, Talbot DC, Tyrell RM, Thompson D. Effect of short-term reduced physical activity on cardiovascular risk factors in active lean and overweight middle-aged men. Metab. 2013;62(3):361-8.

7. Marie-Ludivine CD, Papouin G, Saint-Val P, Lopez A. Effect of adapted karate training on quality of life and body balance in 50-year-old men. Open Access J Sports Med. 2010;4(1):143-50

8. Corrêa LQ, Rombaldi AJ, Silva MC. Atividade física e sintomas do envelhecimento masculino em uma população do sul do Brasil. Rev Bras Med Esporte. 2011;17(4):228-31

9. Andrews M. The seductiveness of agelessness. Ageing Soc. 2000;19(3):301-8.

10. Instituto Brasileiro de Geografia E Estatística (IBGE). Projeção da população do Brasil/censo 2010 [acesso em 2013 set 02]. Disponível em: http//www.ibge.gov.br.

11. Luiz M, Magnanini MMF. A lógica da determinação do tamanho da amostra em investigações epidemiológicas. Cad Saúde Coletiva. 2000;8(2):9-28.

12. Brasil. Ministério da Saúde (MS). Departamento de Análise de Situação de Saúde. Vigilância de Fatores de Risco e Proteção para Doenças Crônicas por Inquérito Telefônico, Vigitel 2009. Brasília, DF: MS; 2010

13. Coqueiro RS, Borges L, Araújo VC, Pelegrini A, Barbosa AR. Medidas auto-referidas são válidas para avaliação do estado nutricional na população brasileira? Rev Bras Cineantropom Desempenho Hum. 2009;11(1):113-9.

14. WHO, World Health Organization. The International Classification of adult underweight, overweight and obesity according to BMI, 2004 [acesso em 2013 jun 23]. Disponível em: http://apps.who.int/bmi/ index.jsp?introPage=intro_3.html.

15. Heinemann LAJ, Saad F, Zimmermann T, Novak A, Myon E, Badia X, et al. The Aging Males Symptoms (AMS) scale: update and compilation of international versions. Health Qual Life Outocomes. 2003;1(1):15.
16. Center for Disease Control and Prevention. Behavioral risk factor suveillance system questionnaire. Altanta: CDC; 1999

17. Pardini R, Matsudo S, Matsudo V, Araújo T, Andrade E, Braggion G, et al. Validation of international physical activity questionnaire (IPAQ): pilot study in brazilian young adults. Med Sci Sports Exerc 1997;29(6):S5-9.

18. Maldonado G, Greenland S. Simulation study of confounder-selection strategies. Am J Epidemiol. 1993;138(11):923-36.

19. WHO. World Health Organization. Global recommendations on physical activity for health, 18-64 years old. 2011 [acesso em 2013 jul 17]. Disponível em: http://www.who.int/dietphysicalactivity/physical-activity-recommendations-18-64years.pdf.

20. Beenackers MA, Kamphuis CBM, Giskes K, Brug J, Kunst AE, Burdof A, et al. Socioeconomic inequalities in occupational, leisure-time, and transport related physical activity among European adults: a systematic review. Int J Behav Nutr Phy. 2012;9:116.

21. Finger JD, Tylleskär T, Lampert T, Mensink GBM. Physical activity patterns and socioeconomic position: the German National Health Interview and Examination Survey 1998 (GNHIES98). BMC Public Health. 2012;12:1079-90

22. Sá-Silva SP, Yokoo, EM, Salles-Costa R. Fatores demográficos e hábitos de vida relacionados com a inatividade física de lazer entre gêneros. Rev Nutr. 2013;26(6):633-45.

23. Mabry RM, Winkler EA, Reeves MM, Eakin EG, Owen N. Correlates of Omani adults' physical inactivity and sitting time. Public Health Nutr. 2013;16(1):65-72.

24. White DK, Tudor-Locke C, Felson DT, Gross KD, Niu J, Nevitt M. Walking to meet physical activity guidelines in knee osteoarthritis: is 10,000 steps enough? Arch Phys Med Rehabil. 2013;94(4):711-7.

25. Moura BP, Marins JCB, Amorim PR. Self selected walking speed in overweight adults: Is this intensity enough to promote health benefits? Apunts Medicina de l'Esport. 2011;46(169):11-15.

26. Matos LDN. Atividade física como uma receita para promoção da saúde cardiovascular, o que é possível fazer em termos práticos. Rev Soc Cardiol Estado de São Paulo. 2012;22(2):14-7.

27. Kim O, Jeon HO. Relationship between obesity, alcohol consumption, and physical activity of male office workers in South Korea. Nurs Health Sci. 2011;13(4):457-62.

28. Schmalz DL. "I feel fat": weight-related stigma, body esteem, and BMI as predictors of perceived competence in physical activity. Obes Facts. 2010;3(1):15-21

29. Napolitano MA, Papandonatos GD, Borradaile KE, Whiteley JA, Marcus BH. Effects of weight status and barriers on physical activity adoption among previously inactive women. Obesity. 2011;19(1):2183-9.

30. Hallal PC, Gomez LF, Parra DC, Lobelo F, Mosquera J, Florindo AA, et al. Lições aprendidas depois de 10 anos de uso do IPAQ no Brasil e Colômbia. J Phys Act Health. 2010;2(S2):S259-64. 